\title{
Assessment of the Potential of Wrist-Worn Wearable Sensors for Driver Drowsiness Detection
}

\author{
Thomas Kundinger ${ }^{1,2,3, *}$, Nikoletta Sofra ${ }^{1}$ and Andreas Riener ${ }^{2,3}$ \\ 1 AUDI AG, 85045 Ingolstadt, Germany; nikoletta.sofra@audi.de \\ 2 Faculty of Computer Science, Technische Hochschule Ingolstadt (THI), 85049 Ingolstadt, Germany; \\ andreas.riener@thi.de \\ 3 Department of Computer Science, Johannes Kepler University (JKU), 4040 Linz, Austria \\ * Correspondence: thomas1.kundinger@audi.de
}

Received: 10 January 2020; Accepted: 10 February 2020; Published: 14 February 2020

check for updates

\begin{abstract}
Drowsy driving imposes a high safety risk. Current systems often use driving behavior parameters for driver drowsiness detection. The continuous driving automation reduces the availability of these parameters, therefore reducing the scope of such methods. Especially, techniques that include physiological measurements seem to be a promising alternative. However, in a dynamic environment such as driving, only non- or minimal intrusive methods are accepted, and vibrations from the roadbed could lead to degraded sensor technology. This work contributes to driver drowsiness detection with a machine learning approach applied solely to physiological data collected from a non-intrusive retrofittable system in the form of a wrist-worn wearable sensor. To check accuracy and feasibility, results are compared with reference data from a medical-grade ECG device. A user study with 30 participants in a high-fidelity driving simulator was conducted. Several machine learning algorithms for binary classification were applied in user-dependent and independent tests. Results provide evidence that the non-intrusive setting achieves a similar accuracy as compared to the medical-grade device, and high accuracies $(>92 \%)$ could be achieved, especially in a user-dependent scenario. The proposed approach offers new possibilities for human-machine interaction in a car and especially for driver state monitoring in the field of automated driving.
\end{abstract}

Keywords: drowsiness detection; driver state; simulator study; physiological measures; machine learning; wearable sensors; automated driving

\section{Introduction}

In its "2025 Roadmap", the EuroNCAP (European New Car Assessment Programme) states that driver state monitoring will be part of the safety assessments by 2020 and listed in the category of primary safety [1]. Around $90 \%$ of all accidents are based on human errors [2], and a considerable part of them occur due to drowsiness. In the USA, for example, $21 \%$ of fatal crashes can be attributed to drowsiness [3]. The risk of an accident is almost four times higher when being in a drowsy physical state [2]. Therefore, reliable driver state monitoring systems are required. By warning an impaired driver already in an early stage, these systems have high potential to increase road safety [1]. Automated driving is expected to have an even higher impact on increasing road safety, as it eliminates potential driver errors. However, the risk factor drowsiness plays and will continue to play a crucial role until the complete rollout of advanced automated driving technologies is achieved. Utilizing the definition of the automation of the driving task provided by the Society of Automotive Engineers (SAE), level 1 (supported driving), 2 (partial automation), and 3 (conditional automation) specifically demand a method to observe the driver's state reliably [4]. In level 1, the driver is supported 
by advanced driver assistance systems (ADAS) that take over either longitudinal or lateral vehicle control in certain situations. In level 2, the driver is required to monitor the system continually and to have the capacity to intercede in a sufficient time period. In level 3, the driver is excluded from monitoring the system yet needs to react sufficiently to a take-over request anytime. At these levels, the driver acts as a fallback for the automated system. However, the decreasing active involvement in driving increases the risk of getting drowsy faster.

\section{Driver Drowsiness Measurement Technologies}

For driver drowsiness detection, various systems and measurement technologies have already been developed that can be summarized into subjective, behavioral, vehicle-based, as well as physiological measures [5,6]. Apart from the measurement of drowsiness in an automotive context, its detection also plays a crucial role and finds use in other areas, such as in the field of aviation or marine operations. Therefore, some examples from other areas with similar detection approaches are listed.

Subjective measures are mainly used as a reference metric for obtaining a definition and ground truth for drowsiness $[7,8]$. These measures include self- and observer ratings. For driver self-ratings, questionnaires such as the Karolinska Sleepiness Scale (KSS) [9], the Epworth Sleepiness Scale (ESS) [10], the Stanford Sleepiness Scale (SSS) [11], or the Visual Analog Scale (VAS) [12] are provided. For observer ratings, experts or trained raters observe the driver either in real-time [13] or by watching videos recorded during an experiment [14]. They evaluate the driver's current drowsiness state with scales that focus on sleep-induced indicators in the facial region $[7,8,14,15]$. Since this type of measurement depends either on the driver himself/herself or an external observer, the usage in a real-world driving scenario is not possible.

Behavior-based methods observe the driver's face with a driver monitoring camera to automatically detect early signs of drowsiness utilizing image processing. This technique has already been investigated for a long time [16,17]. Particularly advantageous is their non-intrusiveness, which makes their usage inside a vehicle very customer friendly. Due to advancements in camera technology in combination with novel approaches in computer vision and image processing, camera-based drowsiness detection has been receiving more and more attention in recent years [18]. These methods evaluate mainly three parameters: eye movements (eye blinking and eye closure activity) via eye-tracking, that was also investigated for usage in maritime operations and aviation [19-21], facial expressions (yawning, jaw drop, brow rise, and lip stretch), and head position (head scaling/nodding) [22]. In particular, many studies focused on the use of machine (deep) learning-based approaches [23-27]. Apart from research, numerous commercial products are available that rely on behavioral measures for drowsiness detection. These range from camera-based methods [28,29] to devices worn behind the ear [30] or glasses [31], that also found their way into the aircraft [32]. However, differing blink frequencies and amplitudes from individual to individual can impact the quality of the observing framework [33]. Moreover, insufficient lighting, sunglasses, or partial visibility of the head by, for example, an unusual sitting position can limit the performance of the monitoring system [22,34].

Vehicle-based systems evaluate driving behavior. The most commonly used measures are Steering Wheel Movement (SWM) and Standard Deviation of Lane Position (SDLP) [22]. In the former, unnatural steering behavior induced by drowsiness is detected by using a steering angle sensor. In the latter, the position of the vehicle in the lane is monitored, and the deviation from the centerline of the lane calculated [35-40]. These measures are non-intrusive and show high potential in detecting drowsiness. This is also reflected in the fact that well-known car manufacturers have been making use of this technology for several years in their series production cars [41-43]. However, their reliability is influenced by driving expertise, geometric, and environmental conditions as well as intoxication [5]. During automated driving, these parameters will be more difficult to evaluate since the automated system controls the vehicle. 
The most commonly investigated physiological-based methods for driver drowsiness detection [22] utilize information based on brain activity (electroencephalography (EEG)) [44-46], cardiac activity (electrocardiography (ECG)) [47-49], ocular activity (electrooculography (EOG)) [50-52], and muscle tone (electromyography (EMG)) [53,54]. Physiological measures are reliable, accurate, and show high potential in differing wakefulness and sleep during driving $[5,55]$. They change in the very early stages of drowsiness compared to behavioral or vehicle-based ones that have the ability to warn the driver in time [5]. However, a major disadvantage is their intrusiveness, e.g., by attaching adhesive electrodes on the subject's chest or scalp. Therefore, new and less intrusive monitoring strategies for recording this kind of signals are required. Different approaches have been proposed to record them in a less or even non-intrusive way inside a vehicle for driver drowsiness detection. For example, experiments were conducted to measure heart rate or ECG for Heart Rate Variability (HRV) analysis via integrated sensors on the steering wheel [56-58]. However, their usage is limited in the context of automated driving since one or even both hands have to touch the steering wheel for a longer time. In the work of Solaz et al., the driver's breathing rate was captured from real-time image recognition. Results show that the kind of clothes influences and reduces system performance [59]. As part of a research project funded by the European Union, bio-sensors were built into car seat fabrics and seatbelts to measure heart rate and respiration [60]. For the approaches mentioned, it is necessary to incorporate sensors in the vehicle additionally. Moreover, the upgrade of existing vehicles would be associated with considerable effort. Apart from research, also on the market, systems for drowsiness detection with physiological signals are available. These devices measure heart rate and Electrodermal Activity (EDA) with a bracelet [61], only EDA with a double ring [62] or Galvanic Skin Response (GSR) with wristband and ring [63]. Lately, devices from the consumer electronics market are being integrated into the vehicle, which could be a solution to the problems mentioned. In this market, especially the trend of fitness tracking and health monitoring in everyday life with wrist-worn smart wearable devices (e.g., smartwatch, fitness tracker) prevails. Due to their cheapness and the fact that they can be worn comfortably like a watch, their usage is expanding continuously, also demonstrating enhancing customer satisfaction and acceptance. The market for wearable gadgets is consistently growing (two-fold growth from 2015 to 2017). In a very recent report, it was stated that the worldwide smartwatch shipments increased by $56 \%$ within 2018 compared to the time period in the previous year [64]. Moreover, it was shown that physiological data from wearables exhibit very high correlations with more advanced measurement devices [65]. Encouraged by the progress in the development of smart wearable devices during the last years, in this work, their suitability in an automotive environment and in particular, in the field of driver drowsiness detection will be investigated. Apart from their use in daily health and fitness monitoring for which these devices were mainly designed, in this way, their scope of use could be further enhanced. This kind of driver-vehicle interaction offers the option of being easily integrated into a vehicle, and existing vehicles can also quickly be upgraded with this technology without having to install additional sensors. Their usage inside a vehicle would enable long-term recording of physiological signals in a non-intrusive and for the driver familiar way.

The rest of the paper is organized as follows: In Section 3, previous work in driver drowsiness detection using wrist-worn wearable devices are discussed, the research gap is highlighted, the contribution, as well as research questions, are presented. Section 4 describes the methodology underlying the work, including simulator study, driver state analysis, feature analysis, data set preparation, and the classification of driver drowsiness. In the results section (Section 5), the findings from feature selection and classification are presented and discussed. This is followed by the conclusion (Section 6), which summarizes the work and core findings.

\section{Previous Work in Driver Drowsiness Detection Using Wrist-Worn Wearable Devices}

Lee et al. utilized the built-in motion sensors of a smartwatch for driver drowsiness detection by evaluating the driving behavior [66]. Twenty subjects participated in a simulator study. Time, spectral, 
and frequency domain features were extracted and mapped to the subject's drowsiness self-ratings. A support vector machine (SVM) classifier reached an accuracy of $98.15 \%$. A similar approach was followed in the work of Lee et al. where accelerometer and gyroscope data in combination with a SVM classifier was used, resulting in an accuracy of $98.8 \%$ [67]. Leng et al. developed a wristband with a photoplethysmogram (PPG) and galvanic skin response (GSR). From data of 20 subjects, five features were extracted, including HRV and respiratory rate, and labeled with self-ratings [68]. A SVM classifier resulted in an accuracy of $98.30 \%$. In the work of Choi et al., a wrist-worn wearable device with sensors for PPG for HRV analysis, GSR, temperature, acceleration, and gyroscope was developed. Twenty-eight people participated in their simulator study. Labels were gathered by analyzing signs of sleepiness in their facial expressions. With a SVM classifier, an accuracy of $98.43 \%$ of accuracy was reached [69]. Lee et al. conducted a simulator study with six participants and combined data from a PPG sensor of a Polar (Polar Electro Oy, Kempele, Finland) smartwatch with ECG data measured with a breast belt. Labels were assigned by evaluating videos of the driver's face and driving behavior. Their classification in the form of recurrence plots resulted in an accuracy of 70\% [47]. The heart rate measurement of a smartwatch was fused with PERCLOS (percentage of eye closure) in the work of Li et al. [70]. A study in a simulated environment with 10 participants was conducted. An accuracy of $83 \%$ was obtained with a SVM classifier.

By utilizing data from smart wearables devices for driver drowsiness detection, promising results were achieved in previous works. However, some devices were specially developed for these studies and are not readily available on the consumer electronics market $[68,69]$. When available on the market, they were combined with another or more intrusive measurement $[47,70]$. Focusing on future automated driving, sensors like accelerometer or gyroscope will be more difficult or in a different way to use since movement patterns from the steering cannot be continuously evaluated for driver state detection $[66,67]$. In view of this, the aim of this work was to investigate and assess the potential and feasibility of using physiological data (HRV) from a wrist-worn wearable device, readily available on the consumer electronics market, as single data source in combination with a common machine learning model for driver drowsiness detection. In further steps and based on the results, the knowledge gained and information provided can then also be applied to develop multimodal systems with a sensor fusion approach and to merge the data of the wrist-worn wearable device with other non-intrusive in-vehicle sensors, e.g., a driver monitoring camera. Within this work, the following research questions (RQ) are addressed:

- RQ1: Is it possible to reliably detect driver drowsiness by using physiological data (HRV) from a wrist-worn wearable device as single data source in combination with a machine learning classifier?

- RQ2: Considering the in-vehicle setting, how do the results of the consumer device differ from a more intrusive medical-grade device?

- RQ3: How do the results differ in the case of user-dependent vs. user-independent tests?

\section{Methodology}

To examine the feasibility of our proposed approach for detecting driver drowsiness, the methodology, as presented in Figure 1, was applied. The three main stages, including simulator study, feature extraction, and machine learning, will be described in more detail in the following sections. 


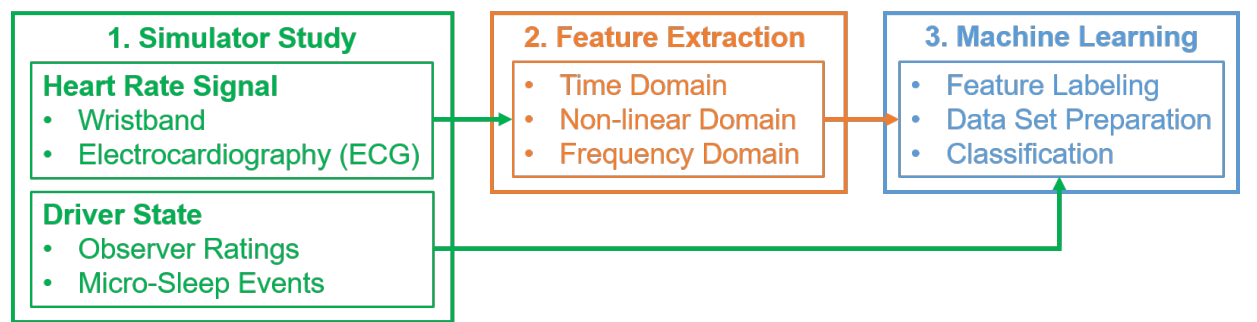

Figure 1. Overview of the methodology for the proposed drowsiness detection system. ECG: Electrocardiography.

\subsection{Simulator Study}

To record measurements of heart rate data and driver state, a user study in a simulator was conducted, which will be explained in the following sections. A more detailed description of the study can be found in the work of Kundinger et al. [71]. The following summary focuses on contextual information and mainly on those parts of the user study that are relevant to this work.

\subsubsection{Simulator}

The experiment was carried out in a high-fidelity driving simulator at Technische Hochschule Ingolstadt (THI, Ingolstadt, Germany). The course chosen was a monotonous track (closed loop with a length of around $13 \mathrm{~km}$ ) in the form of a three-lane highway with little traffic (see Figure 2). The average temperature in the test vehicle was $25^{\circ} \mathrm{C}$. No radio or music was played during the drive.

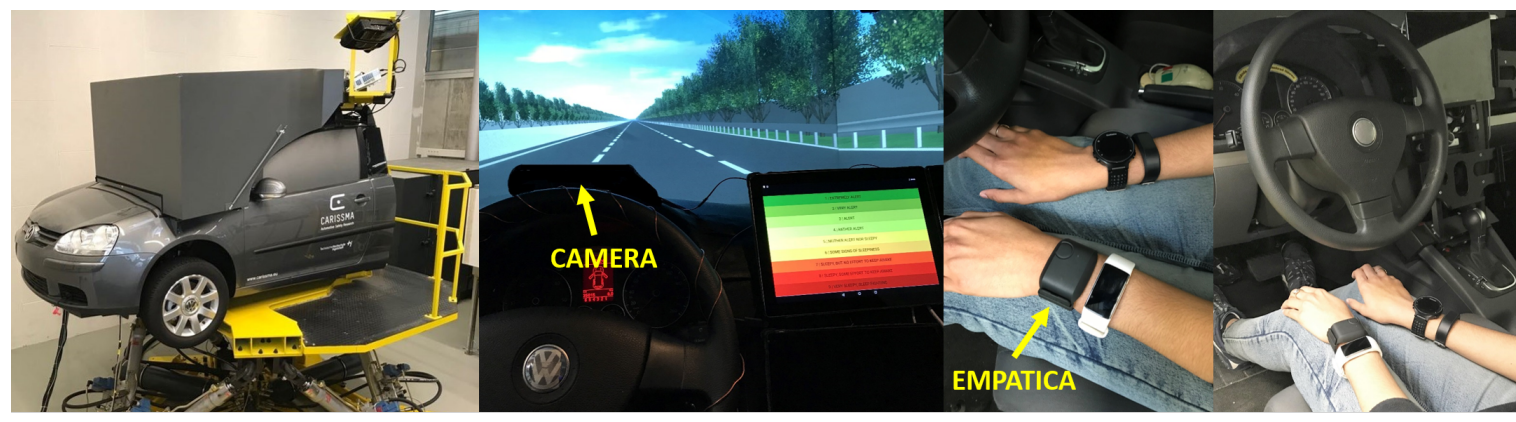

Figure 2. (Left) High-fidelity driving simulator (Hexapod system) as used in the study; (Center) Study setup with camera mounted on top of cockpit; (Right) Wearable devices on wrist of participant.

\subsubsection{Participants}

Thirty subjects participated in the study, students, or recruited via an advertisement in a local newspaper. Concerning a report from the Sleep Health Foundation that contains recommendations about sleep requirements over the life span, subjects were divided into two age groups [72]. Fifteen younger ( 6 female; 9 male; mean $(M)=22.87$ years; standard deviation $(\mathrm{SD})=1.81$ years) subjects were chosen in the age range $20-25$ and 15 older subjects ( 8 female; 7 male; $M=67.60$ years; $\mathrm{SD}=1.88$ years) in the range $65-70$. For their participation, each subject received $€ 35$. The participation requirements were a valid driving license, self-reported good health, no sleep disturbances, and no restrictions on driving ability. They were not allowed to consume caffeinated drinks within five hours before the study.

\subsubsection{Study Procedure}

In the beginning, the subjects got instructions and filled out a questionnaire. The first part contained some basic demographic questions and queried details about sleeping behavior and health. Secondly, subjects were asked to answer the questions of the ESS to measure their daytime sleepiness [10]. This was followed by a 10-min manual test drive to become familiar with the simulator. 
Then, the 45-min automated ride began. The speed limit for the automated drive was set to $110 \mathrm{~km} / \mathrm{h}$. The simulation car traveled with autonomous cruise control (ACC). It automatically accelerated and decelerated, depending on the traffic. A total distance of $73.7 \mathrm{~km}$ was covered during the partially automated drive. To reproduce SAE level-2 driving [4], drivers were asked to monitor the driving condition continuously and to be prepared to assume control in the event of a take-over request. In the context of this study, take-overs were not necessary as they would probably have interfered with the development of drowsiness. The request to observe the driving environment had the objective to maintain the driver's concentration, inducing a more rapid development of drowsiness. Participants were asked not to use their mobile phone, not to eat or drink and abstain from chewing gum, or to nod off. They were not allowed to carry out any secondary tasks. Moreover, the participants drove manually (in randomized order) under similar conditions. The study was conducted at 9 a.m., 1:30 p.m., and 5:30 p.m. An almost equal number of participants were available at each time (morning: 9, afternoon: 11, evening: 10).

The corresponding results of the questionnaires are published and can be found in the work of Kundinger et al. [71].

\subsubsection{Measurement Technique}

In terms of the wrist-worn wearable consumer device, heart rate signals were recorded utilizing an Empatica E4 wristband (Empatica Inc., Boston, MA, USA) (further referenced as "wristband") [73]. The Empatica E4 wristband records inter-beat intervals (IBIs), the time between two successive and individual heartbeats, for calculating the instantaneous heart rate that will be later used in HRV analysis. Another commonly used description of the IBIs is RR intervals, which is the time elapsed between two successive $\mathrm{R}$ waves, i.e., two heartbeats, of the QRS complex on the ECG. This IBI sequence is received from the PPG/BVP (blood volume pulse) signal with a sampling frequency of $64 \mathrm{~Hz}$. McCarthy et al. checked the validity of the Empatica E4 wristband against clinical standard gears in recognizing the anomalies in a heartbeat and found a comparable data quality of $85 \%$ between these devices [74]. A 3-channel ECG measurement device (further referenced as "ECG"), the Faros from Bittium (Bittium Corporation, Oulu, Finland) [75], served as the medical-grade device and reference for the wristband. The ECG data was recorded with the maximum possible sampling frequency of $1000 \mathrm{~Hz}$. With five adhesive electrodes, it was attached to the subject's upper body. RR intervals were stored for HRV analysis. By performing HRV analysis on ECG or PPG signals, the activity of the Autonomic Nervous Systems (ANS) can be obtained [76]. This activity alters with stress and drowsiness [77]. The two major components of the ANS are the sympathetic and parasympathetic nervous systems. The decrease of parasympathetic activity and increase of sympathetic activity is associated with the vigilance of a person. The increase in parasympathetic activity and the decrease in sympathetic activity indicates relaxation [77]. The normalized Low-Frequency (LF) band power of HRV follows the dominance of sympathetic activity, whereas High-Frequency (HF) power is associated with parasympathetic activity [78]. Therefore, investigating ANS activity may help to get deeper insights in a driver's drowsiness [79]. Besides physiological signals, videos of the driver's face were recorded, which were used to assess the driver's current drowsiness state. Therefore, a camera was mounted on top of the cockpit and directed towards the driver's face (see left part of Figure 2).

Further data (not evaluated in this work) were recorded: Physiological data with three additional wearable devices (Polar A370 (Polar Electro Oy, Kempele, Finland), Garmin Vivosmart 3 (Garmin Ltd., Schaffhausen, Switzerland), Garmin Forerunner 235 (Garmin Ltd.)), driving behavior-related parameters and drowsiness self-ratings (see Kundinger et al. [71]).

\subsection{Driver State Analysis}

In order to obtain a reliable and valid ground truth of drowsiness for supervised machine learning, a two-stage process with a combination of video ratings and image processing was applied. 


\subsubsection{Video Ratings}

Observer ratings of the driver's facial expressions and behaviors were collected offline after the study. The 45-min partially automated ride was split into nine intervals of five min length. From each 5-min interval, one minute was extracted to be rated by the observer. Sandberg et al. found that most indicators of driver sleepiness can be observed for intervals of $60 \mathrm{~s}$ or longer to obtain reasonable signs of a driver's sleepiness state [80]. The order of the extracted segments of one minute length was randomized per participant with the aim of eliminating the time dependency of the single segments. Video segments at the end of a participant's drive would probably be rated higher compared to ones in the beginning. To increase the reliability of the results, two trained individuals rated all videos separately. Following that, the segments with inconsistent ratings were evaluated and discussed by both raters, and a joint rating was set. The obtained rating of the extracted minute from the respective 5-min interval represents the entire interval with the assumption that the drowsiness state does not change abruptly, but rather more slowly. Weinbeer et al. published the scale that was used for the observer ratings. This scale categorizes drowsiness into six levels ranging from level 1 (not drowsy) to level 6 (extremely drowsy) (see Table 1) [13].

Taking into account the 30 subjects and the 45 -min partially automated ride, a total of $270 \mathrm{~min}$, i.e., ratings, would have been available for evaluation. However, for some subjects, problems with the video recording occurred, or the face was only partially visible in the video, for example, due to an unusual seating position. These segments were removed so that in the end, $244 \mathrm{~min}$ were evaluated. By both raters, the same decisions were made in 191 of 244 cases, which correspond to a percentage of $78.28 \%$. Inter-rater reliability in the form of Cohen's Kappa resulted in a value of 0.69 , which represents "substantial agreement" following the classification of Landis and Koch [81].

Table 1. Scale for video ratings with indicators per level [13].

\begin{tabular}{cl}
\hline $\begin{array}{c}\text { Drowsiness Level } \\
\mathbf{1} \\
\text { not drowsy }\end{array}$ & $\begin{array}{l}\text { appearance of alertness present; normal facial tone; normal fast eye blinks; } \\
\text { short ordinary glances; occasional body movements/gestures }\end{array}$ \\
\hline $\begin{array}{c}\mathbf{2} \\
\text { slightly drowsy }\end{array}$ & $\begin{array}{l}\text { still sufficiently alert; less sharp/alert looks; longer glances; slower eye blinks; } \\
\text { first mannerisms as: rubbing face/eyes, scratching, facial contortions, moving } \\
\text { restlessly in the seat }\end{array}$ \\
\hline $\begin{array}{c}\text { 3 } \\
\text { moderately drowsy }\end{array}$ & $\begin{array}{l}\text { mannerisms; slower eye lid closures; decreasing facial tone; glassy eyes; staring at } \\
\text { fixed position }\end{array}$ \\
\hline $\mathbf{4}$ & $\begin{array}{l}\text { eyelid closures (1-2 s); eyes rolling sideways; rarer blinks; no proper focused eyes; } \\
\text { decreased facial tone; lack of apparent activity; large isolated or } \\
\text { punctuating movements; }\end{array}$ \\
\hline $\begin{array}{c}\text { drowsy } \\
\text { very drowsy }\end{array}$ & $\begin{array}{l}\text { eyelid closures (2-3 s); eyes rolling upward/sideways; no proper focused eyes; } \\
\text { punctuating movements; }\end{array}$ \\
\hline $\mathbf{6}$ & $\begin{array}{l}\text { eyelid closures (4 s or more); falling asleep; longer periods of lack of activity; } \\
\text { movements when transition in and out of dozing; }\end{array}$ \\
\hline
\end{tabular}

\subsubsection{Detection of Micro-Sleep Events through Image Processing}

In addition to the observer ratings, the recorded video data was evaluated through image processing. As the raters only assessed one minute in each 5-min interval of the 45-min ride, drowsiness indicators in the remaining four minutes could not be considered for the final rating. Concerning the drowsiness scale, this would have been particularly critical if a micro-sleep event had taken place within the non-rated minutes since levels 4 to 6 include the specific eyelid closure time as a drowsiness indicator. Therefore, all detected events with an eye closure of at least one second or longer were used, as well as the respective eyelid closure duration in order to assign the appropriate level of drowsiness. With this additional step, the observer ratings could be cross-checked and enhanced. 
In total, 201 micro-sleep events were detected for 14 out of 30 subjects. All events detected were manually double-checked based on the corresponding frame numbers in the video file. The events were split, as shown in Table 2 to be assigned directly to drowsiness levels 4 to 6 on the scale used. As the scale (see Table 1) does not take into account eyelid closures between 3 and 4 s (level 5: 2-3 s and level 6: 4 s or more), these have been added to level 5 (see Table 2).

Table 2. Categorization and allocation of the 201 detected micro-sleep events to the corresponding level of drowsiness based on the eyelid closure time.

\begin{tabular}{lrc}
\hline Drowsiness Level & Eyelid Closure Time & Micro-Sleep Events \\
\hline 4: drowsy & $1 \leq$ seconds $<2$ & 89 \\
5: very drowsy & $2 \leq$ seconds $<4$ & 69 \\
6: extremely drowsy & seconds $\geq 4$ & 43 \\
\hline
\end{tabular}

After receiving video ratings and micro-sleep events, both measures were combined. For each subject, the ratings for the 5-min intervals with micro-sleep events were adjusted if necessary. A change in the drowsiness level was done for 23 out of the 244 received ratings. The drowsiness level was corrected 20 times upwards and one time downwards. Two new ratings could be gained, giving a total of 246 ratings (see Table 3 ).

Table 3. Adjustment of observer ratings after integration of detected micro-sleep events with number of occurrences for each case; n.a.: no observer rating available.

\begin{tabular}{ccc}
\hline Observer Rating & Adjusted Rating & Number of Occurrences \\
\hline 1 & 4 & 1 \\
\hline 2 & 4 & 3 \\
2 & 5 & 4 \\
2 & 6 & 1 \\
\hline 3 & 4 & 2 \\
3 & 5 & 5 \\
\hline 4 & 5 & 3 \\
\hline 5 & 6 & 1 \\
\hline 6 & 4 & 1 \\
\hline n.a. & 6 & 2 \\
\hline
\end{tabular}

Since the main focus of this work is on the assessment of the potential of wrist-worn wearable sensors for driver drowsiness detection, the simple case of binary classification of drowsiness ("not drowsy" vs. "drowsy") is considered. In further steps and with the knowledge acquired in this work, the classification of sleepiness can also be extended to a multi-class problem. Therefore, the six drowsiness levels of the considered scale were divided into two groups. The "non-drowsy" class contains levels 1 to 3 (not drowsy, slightly drowsy, moderately drowsy) whereas the "drowsy" class covers levels 4 to 6 (drowsy, very drowsy, extremely drowsy). Table 4 shows the distribution of the number of grouped ratings on the two classes before and after the adjustment with micro-sleep events. A class imbalance is apparent.

Table 4. Distribution of number of video ratings in absolute numbers across grouped drowsiness levels before and after integration of micro-sleep events.

\begin{tabular}{lcc}
\hline Class & Video Ratings & $\begin{array}{c}\text { Video Ratings + } \\
\text { Micro-Sleep Events }\end{array}$ \\
\hline non-drowsy (level 1-3) & 212 & 196 \\
drowsy (level 4-6) & 32 & 50 \\
\hline
\end{tabular}


In Figure 3, the localization in time of the number of ratings for both the "non-drowsy" and "drowsy" class after the integration of micro-sleep events is presented. This shows that the number of "drowsy" ratings increased almost linearly across all subjects up to a driving time of $30 \mathrm{~min}$. In contrast, the number of "non-drowsy" ratings decreased in the same time interval. From minutes 30 to 40 , it shows exactly the opposite, and a trend similar to the one at the beginning of the drive can be observed in the last five minutes. From this it can be deduced that drowsiness increased on average across all subjects up to minute 30 , and decreased from minutes 30 to 40 . Towards the end, the level of drowsiness rose again.

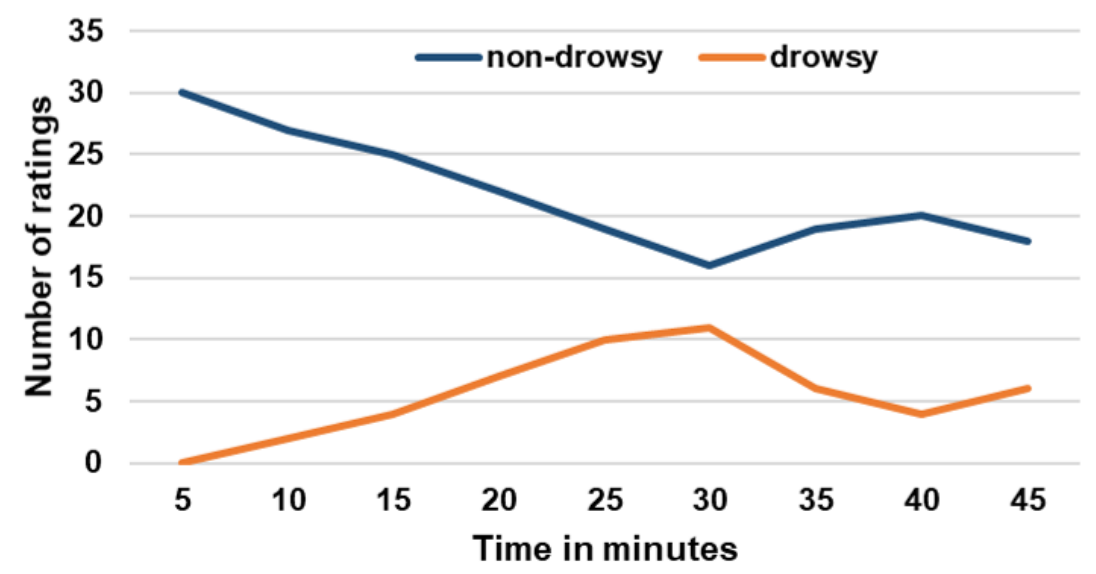

Figure 3. Localization in time of number of ratings for both the "non-drowsy" and "drowsy" class.

\subsection{Feature Extraction and Data Set Preparation}

Feature extraction and data set preparation for machine learning was performed with MATLAB (The MathWorks, Inc., Natick, MA, USA) (version 2018a). The different sampling rates of the two devices were not adjusted because this work aims to compare the usage of data from a consumer and a medical-grade device. Since three channels were recorded with the ECG measuring device, but only one and the same channel was used for all participants for further analysis, the three channels of all subjects were visually inspected in terms of data quality and possible artifacts, e.g., undetected RR peaks in the ECG pattern, with the Kubios HRV analysis software [82]. Finally, the RR peaks of Channel 1 were applied in further analysis in raw format. In terms of Empatica E4 wristband, it should be mentioned that the wristband uses an algorithm during the recording of the data and thus the processing of the PPG/BVP signal that already filters and removes false peaks due to noise (e.g., motion artifacts) [83]. For this reason, the raw data of the wristband was used for the following analyses and is not further filtered or preprocessed. IBIs from wristband and ECG were processed in time, frequency, and non-linear domain for HRV analysis. In several previous works, HRV was applied for driver drowsiness detection [69,76,84]. HRV features were extracted from 5-min windows of the signal with reference to the Task Force of the European Society of Cardiology and the North American Society of Pacing and Electrophysiology that suggests 2-5 min windows for the analysis of frequency-domain features [78]. A sliding window with a 2-s increment was used for generating the feature vectors (see Figure 4). In the frequency domain, Power Spectral Density (PSD) was computed with Lomb-Scargle periodogram, a non-parametric technique that does not require any earlier information of the signal parameters. The advantage of utilizing Lomb-Scargle periodogram over other non-parametric techniques like Welch is that no interpolation is needed if the signals are non-periodic. From three different frequency bands of PSD features were extracted: very low frequency (VLF) band (0-0.04 Hz), low frequency (LF) band $(0.04-0.15 \mathrm{~Hz})$ related to the sympathetic activity, and high-frequency $(\mathrm{HF})$ band $(0.15-0.4 \mathrm{~Hz})$ related to the parasympathetic activity of ANS [76]. Features in the non-linear domain were calculated with Poincare plots. The following 26 features were extracted (abbreviations in brackets): 
- Time-domain features: Mean RR interval length (meanRR), maximum RR interval length (maxRR), minimum RR interval length (minRR), range of RR interval length (rangeRR), standard deviation of RR interval lengths (SDNN), mean of 5-min standard deviation of RR intervals (SDANNIndex), maximum heart rate (maxHR), minimum heart rate (minHR), average heart rate (meanHR), standard deviation of heart rate (SDHR), square root of the mean squared difference of successive RR intervals (RMSSD), number of interval differences of successive RR intervals greater than $50 \mathrm{~ms}$ (NN50), percentage of successive/adjacent RR intervals differing by $50 \mathrm{~ms}$ (pNN50);

- Frequency-domain features: Very low frequency power (VLF), low frequency power (LFpower), high frequency power (HFpower), total power (Totalpower), percentage value of very low frequency power (pVLF), percentage value of low frequency power ( $\mathrm{pLF})$, percentage value of high frequency power (pHF), normalized low frequency power (LFnorm), normalized high frequency power (HFnorm), ratio of low and high frequency (LFHF_ratio);

- Non-linear domain features: Standard deviation of instantaneous (short term) beat-to-beat R-R interval variability (SD1), standard deviation (SD) of the long term R-R interval variability (SD2), ratio of standard deviation 1 and standard deviation 2;

After extraction, features were labeled for supervised machine learning (see Figure 4). In total, data from 27 out of 30 subjects were available for feature extraction from the wristband. To provide comparability, the missing three subjects were not considered in the case of the ECG. Overall, the number of "non-drowsy" and "drowsy" instances for the wristband are 14,627 and 3987 and for ECG 24149 and 5845. It becomes clear that ECG contains more instances, which is due to a more accurate, higher-resolution measurement compared to the wristband.

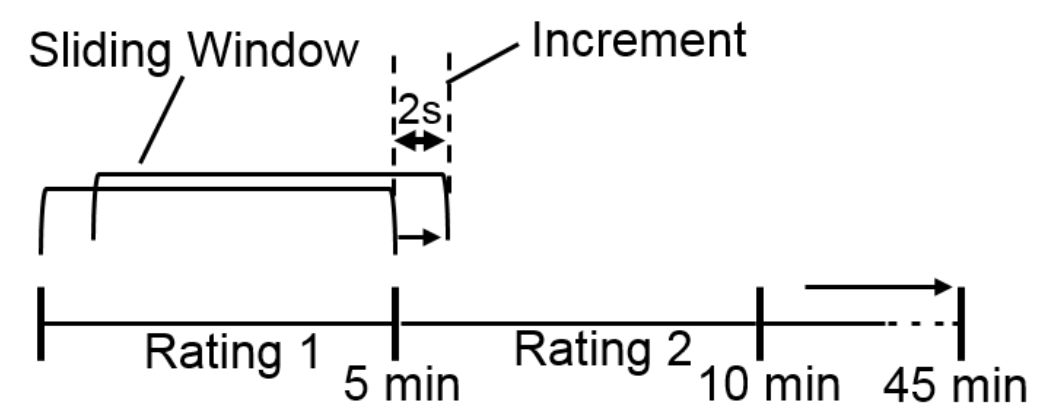

Figure 4. Sliding window approach for feature extraction and labeling exemplary for time intervals 1 and 2.

\subsection{Classification of Driver Drowsiness}

The ability to generalize for new users is a crucial issue for the establishment of systems for driver activity recognition. Thereby, the problem of inter-driver variance has to be taken into account, because physiological signals within persons, in our case, drivers of an automated vehicle, can differ to a great extent [85]. We apply a User-Independent Test (UIT) to deal with this issue. In the UIT, a Leave-One-Subject-Out Cross-Validation (LOSOCV) is performed. The data set for each subject is treated as testing data once. Since, in the present case, data from 27 participants were collected, in each LOSOCV-iteration, 26 participants are used for training and the remaining 27th for testing. The prediction results are then averaged over all subjects (see Figure 5). In comparison to the UIT, a User-Dependent Test (UDT) will be performed additionally in the form of 10-fold stratified Cross-Validation (CV) to obtain the overall classification accuracy and to decrease the effect of inter-driver variance. Stratified cross-validation was utilized because each fold reflects the class distribution in the original data set. In terms of our classification problem and the present class imbalance, this ensures the same proportion of drowsy and non-drowsy samples in each cross-validation run. Moreover, it reduces both bias and variance compared to regular k-fold cross-validation, where the data set is only randomly divided into $\mathrm{k}$ folds [86]. 


\subsubsection{Class Balancing and Feature Selection}

To counteract class imbalance, the number of instances in the two classes has been adjusted using the "Synthetic Minority Oversampling Technique (SMOTE)" [87]. During LOSOCV and 10-fold cross-validation, SMOTE was applied to the training set in each iteration before the classifier was trained (see Figure 5).

To reduce the feature space, feature selection on each training set was performed in each iteration step after oversampling. For this purpose, Correlation-based Feature Subset Selection (CFSS) was applied. CFSS searches for a subset of features that is highly correlated to the output class, yet its features unrelated to each other. Thereby, feature-to-feature correlation is reduced, and feature-to-class correlation increased. The criterion is defined using the Pearson correlation coefficient. CFSS is not a search method; rather, it proposes a metric to evaluate a certain feature subset. Best-first was used as search algorithm. The predictive ability of each feature is used to evaluate the worth of a subset, in consideration of the redundancy between all features. [88]. Depending on the features in the obtained subset, in each iteration, the corresponding features were adjusted in the corresponding test data set (see Figure 5).

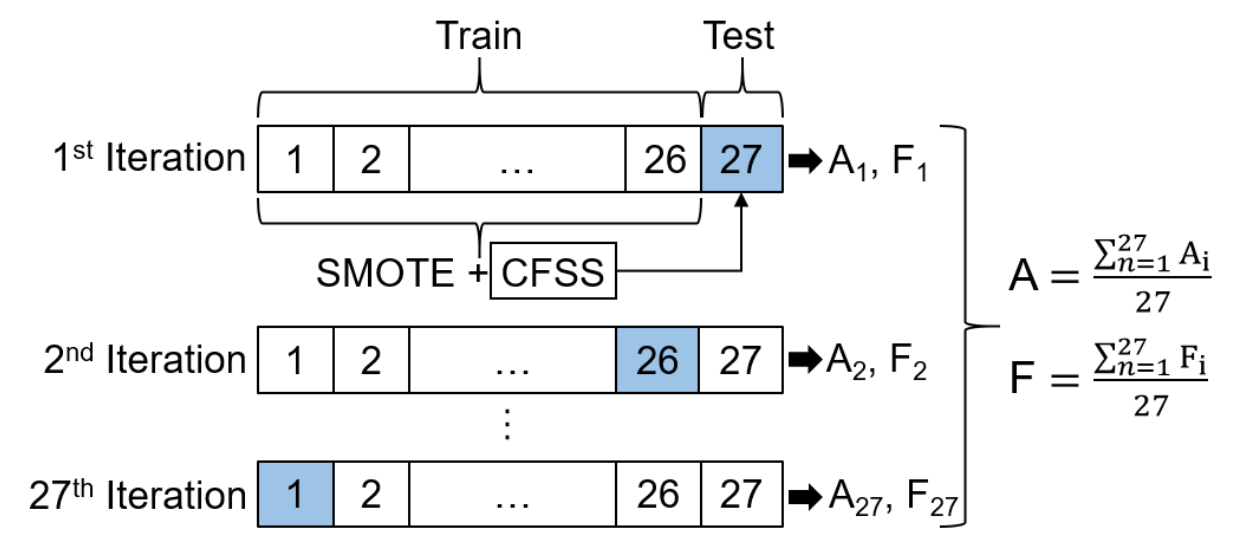

Figure 5. Graphic representation of Leave-One-Subject-Out Cross-Validation (LOSOCV) for 27 subjects: Oversampling of minority class in training set in each iteration with Synthetic Minority Oversampling Technique (SMOTE); Selection of best feature subset with Correlation-based Feature Subset Selection (CFSS) in training set and adjusting of features in testing set; calculation of average accuracy (A) and F-measure (F) (separately for both classes) across all iterations.

\subsubsection{Performance Measures}

Concerning performance measures, accuracy might be as one of the traditional measures suitable but is not when dealing with unbalanced data because its focus is more on the majority classes than on the minority ones [89]. Thus, F-measure will be used additionally. For both UDT and UIT, accuracy and F-measure were calculated and averaged across all iterations (see Figure 5). With regard to the presented binary classification problem, it is, of course, important to correctly detect when the driver is in a drowsy state. From the customer's point of view, however, it is also crucial to correctly detect when the driver is in a non-drowsy state, so as not to irritate with unnecessary drowsiness warnings. Concerning a standard confusion matrix with the values for "True Positive (TP)", "True Negative $(\mathrm{TN})$ ", "False Positive (FP)", and "False Negative (FN)", the formula for F-measure (see Equation (1)) does not take the True Negative (TN) values in account.

$$
F-\text { measure }=\frac{2 T P}{2 T P+F P+F N}
$$

In the presented case, the correctly classified instances of the negative class, representing "drowsy", would not be considered. For this reason, at each cross-validation iteration, the value for F-measure 
is calculated per class. For the "non-drowsy", i.e., positive class, this is further referenced as F1 and for the "drowsy", i.e., negative class as F2. An average value is then presented for both F1 and F2 across all subjects. Therefore, F2 is the crucial measure for the detection of drowsiness, since its value represents how many "drowsy" instances were correctly classified as "drowsy".

\section{Results}

The machine learning procedure, including oversampling and feature selection, was performed with the Weka machine learning library implemented in Java [90].

\subsection{Selected Features}

The feature selection procedure using CFSS was performed on the training data. In the next step, the features in the testing data were adjusted accordingly. This was done before the actual training and testing the machine learning model. Table 5 presents all selected feature subsets in CFSS for UDT and UIT for both devices. The total number of available subsets equals the number of iterations in each cross-validation: 10 for UDT (10-fold stratified CV) and 27 for UIT (LOSOCV with 27 subjects).

Table 5. Selected Feature Subsets in CFSS for User-Dependent Test (UDT) (10 iterations in 10-fold Cross Validation (CV)) and User-Independent Test (UIT) (27 iterations in LOSOCV) with number of occurrences for wristband and ECG.

\begin{tabular}{cl}
\hline & UDT \\
\hline \multirow{3}{*}{ Wristband } & $1 \times$ meanRR, meanHR \\
& $1 \times$ meanRR, meanHR, RMSSD \\
& $3 \times$ maxRR, minRR, maxHR, minHR \\
& $2 \times$ maxHR, minHR \\
ECG & $5 \times$ maxRR, minRR \\
& \multicolumn{1}{c}{ UIT } \\
\hline & $5 \times$ meanRR, meanHR, SD1 \\
& $1 \times$ meanRR, maxRR, SD1, LFpower \\
& $1 \times$ meanRR, maxRR, meanHR \\
& $5 \times$ meanRR, meanHRV, RMSSD \\
& $9 \times$ meanRR, meanHR \\
& $1 \times$ meanRR, meanHR, HFpower \\
& $1 \times$ meanRR, meanHR, pNN50, SD1 \\
& $1 \times$ meanRR, meanHR, RMSSD, LFpower \\
& $1 \times$ meanRR, meanHR, SD2 \\
& $1 \times$ meanRR, meanHR, Totalpower \\
& $1 \times$ meanRR, minHR, meanHR, RMSSD, NN50 \\
\hline ECG & $8 \times$ maxRR, minRR, maxHR, minHR \\
& $5 \times$ maxRR, minRR, rangeRR, maxHR, minHR \\
& $3 \times$ maxRR, maxHR \\
& $1 \times$ maxHR, minHR \\
& $6 \times$ minRR, minHR \\
& $4 \times$ maxRR, minRR \\
\hline &
\end{tabular}

When looking closer at the selected feature subsets for the UDT, it can be seen that they consist only of time-domain features. However, the subsets of both devices do not contain any identical features. In the case of the wristband, a total of five different features are selected. Each subset includes meanRR and meanHR. In the case of ECG, maxRR and minRR appear in eight subsets. Furthermore, maxHR and minHR were selected. The low number of subsets can be related to the fact that the data of all subjects were randomized and evenly divided into ten folds in a stratified way to counter inter-driver variance between the subjects. 
In contrast, when looking at the selected feature subsets for UIT and comparing them with those for the UDT, 11 different feature subsets were selected with data from the wristband, which are almost four times as many as for UDT. In addition to time-domain features, in CFSS also features from the frequency- and non-linear domain were selected in the feature subsets. It becomes clear that the inter-driver variance has an influence on the choice of features if only a single person is removed from the data set. This can also be recognized for ECG where twice as many feature subsets exist but, as for the UDT, containing again only features from the time-domain and therein $\mathrm{min} / \mathrm{max}$ values of the RR and HR signals.

In general, for both the UDT and UIT, the majority of selected feature subsets for wristband and ECG mainly consist of time-domain features. The importance of the features for the present case can be ranked in descending order as follows: time-domain, frequency-domain, non-linear domain.

\subsection{Classification Results}

Several classifiers of the Weka machine learning library were applied with the data collected during the simulator study. The models were not fine-tuned, but the default parameter values used, that were preset in the Weka machine learning library [90]. Models from different categories were compared with each other in terms of their performance in the presented classification problem. From the category of tree classifiers, Random Forest (RF) (100 trees), Random Tree (RT), and Decision Stump (DS) were chosen. In terms of rule-based classifiers, a Decision Table (DT) (search algorithm: Best first, evaluation measure: root-mean-square error (RMSE)) was applied. The K-Nearest Neighbor (KNN) algorithm (no distance weighting; number of neighbors: 1, search algorithm: brute-force, distance function: Euclidean) from the group of lazy learners and from the group of Bayesian classifiers a Bayesian Network (BN) (estimator: simple estimator, search algorithm: K2), and Naive Bayes (NB) classifier were tested. A Support Vector Machine (SVM) classifier (kernel: polynomial, C: 1) represented a function-based-classifier. A Multilayer Perceptron (MLP) (batch size: 100, hidden layers: (number of features + number of classes) $/ 2$, learning rate: 0.3 , momentum: 0.2 ) from the group of neural networks was applied. For each of the models presented, the exact same procedure for testing was carried out, i.e., a 10-fold stratified CV in the UDT and a LOSOCV in the UIT, as described above.

Table 6 shows the results of UDT and UIT, for both devices and all models tested, with the respective values for accuracy and F-measure. Focusing on the accuracy of the UDT, it can be seen that ECG data produced better results on all tested classifiers except NB. These differences are, in some cases, more pronounced as in BN, SVM, DS, and DT, but for KNN, RF, and RT, the difference is only a few percentage points. For the wristband, the highest accuracy of $92.13 \%$ was achieved with KNN, $91.58 \%$ with RF, and 90.02\% with RT. For ECG, the classifiers RF and RT performed best with an accuracy of $97.37 \%$, as well as BN with $96.85 \%$ and DT with $91.18 \%$. In terms of F-measure, it is noticeable that the values for F1 and F2 diverge more in the wristband. Looking at RF and RT, the values for F1 are 0.94 and 0.93 , and the values for F2 are 0.82 and 0.79. For ECG, these are 0.98 and 0.94 each. In general and concerning F1, the values of the wristband are slightly lower but comparable to ECG. Except for DS and MLP, which has higher values for F2 than for ECG, the differences between the F1 and F2 are larger. redFor ECG, the values for F1 and F2 are very high and at a similar level for $\mathrm{BN}, \mathrm{KNN}, \mathrm{RF}, \mathrm{RT}$, and DT. This speaks for a low number of false positives and false negatives and for an equally satisfying classification in both classes. The assessment of drowsy instances as really drowsy has shown to be more difficult for the models when working with data from the wristband what can be referred to lower values for F2 and a higher number of false negatives, compared to ECG.

With regard to UIT and except SVM (65.64\% accuracy), overall lower classification accuracies were achieved when compared to UDT. In addition to NB $(66.74 \%)$, SVM $(65.64 \%)$, and MLP $(25.84 \%)$ yielded better results with data from the wristband. All other models achieved higher results with HRV from ECG data. However, the differences between the two devices are not as high as in the UDT case. DS achieved the highest accuracy with $73.39 \%$ and was the only model $>70 \%$. Regarding ECG data, the classifiers NB (41.84\%), SVM (40.01\%), and MLP (25.84\%) did not reach the threshold of 
$50 \%$. In terms of accuracy, DS scored the best with ECG at 78.94\%. Focusing on the values of F1 and F2, it is noticeable that in F1 for both devices, the values are lower compared to the UDT. In the UIT, the maximum F1 score for both the wristband and ECG was achieved with DS (0.82). In contrast, values for F2 are significantly lower. DS achieved a maximum of 0.65 with data from the wristband and DT with ECG data 0.37. In general, the number of false negatives in the "drowsy" class is way higher than the number of false positives in the "non-drowsy" class, which resulted in the low values for F2. To get a better impression of the classification results, Table 7 shows exemplary UIT classification results for four individual subjects $(3,4,21$, and 27) and five selected classifiers (BN, KNN, RF, RT, and DT) for wristband and ECG. The values for F2 show again that the classification of "drowsy" instances is more challenging than the "non-drowsy" ones. Generally speaking, it also becomes clear that the performance depends very much on the individual subject, which reflects the strong influence of the inter-driver variance. Depending on the model, but also on the type of data, strong fluctuations in performance exist already within this small extract from the data set.

Table 6. Classification results with performance measures for UDT/UIT, Wristband/ECG and for all tested classifiers. The abbreviations stand for accuracy (A), F-measure for "non-drowsy" class (F1) and F-measure for "drowsy" class (F2). The best performing models are printed in boldface.

\begin{tabular}{ccccccc}
\hline UDT & \multicolumn{3}{c}{ Wristband } & & & ECG \\
\hline Model & A & F1 & F2 & A & F1 & F2 \\
\hline BN & 79.59 & 0.86 & 0.62 & $\mathbf{9 6 . 8 5}$ & $\mathbf{0 . 9 8}$ & $\mathbf{0 . 9 2}$ \\
NB & 68.39 & 0.79 & 0.32 & 53.88 & 0.66 & 0.26 \\
SVM & 29.27 & 0.72 & 0.32 & 54.39 & 0.66 & 0.28 \\
KNN & $\mathbf{9 2 . 1 3}$ & $\mathbf{0 . 9 5}$ & $\mathbf{0 . 8 3}$ & 97.34 & 0.98 & 0.94 \\
RF & $\mathbf{9 1 . 5 8}$ & $\mathbf{0 . 9 4}$ & $\mathbf{0 . 8 2}$ & $\mathbf{9 7 . 3 7}$ & $\mathbf{0 . 9 8}$ & $\mathbf{0 . 9 4}$ \\
RT & $\mathbf{9 0 . 0 2}$ & $\mathbf{0 . 9 3}$ & $\mathbf{0 . 7 9}$ & $\mathbf{9 7 . 3 7}$ & $\mathbf{0 . 9 8}$ & $\mathbf{0 . 9 4}$ \\
DS & 77.60 & 0.86 & 0.36 & 81.36 & 0.89 & 0.21 \\
DT & 80.61 & 0.87 & 0.65 & $\mathbf{9 7 . 1 8}$ & $\mathbf{0 . 9 8}$ & $\mathbf{0 . 9 3}$ \\
MLP & 61.94 & 0.69 & 0.46 & 64.43 & 0.71 & 0.30 \\
\hline UIT & & Wristband & & & ECG & \\
\hline Model & $\mathbf{A}$ & F1 & $\mathbf{F 2}$ & $\mathbf{A}$ & F1 & F2 \\
\hline BN & 57.77 & 0.69 & 0.19 & 70.86 & 0.77 & 0.10 \\
NB & 66.74 & 0.80 & 0.57 & 41.84 & 0.63 & 0.20 \\
SVM & 65.64 & 0.79 & 0.26 & 40.01 & 0.61 & 0.28 \\
KNN & 55.44 & 0.71 & 0.12 & 65.71 & 0.75 & 0.14 \\
RF & 62.36 & 0.74 & 0.20 & 70.64 & 0.79 & 0.13 \\
RT & 63.16 & 0.72 & 0.19 & 68.88 & 0.76 & 0.21 \\
DS & $\mathbf{7 3 . 3 9}$ & $\mathbf{0 . 8 2}$ & $\mathbf{0 . 6 5}$ & $\mathbf{7 8 . 9 4}$ & $\mathbf{0 . 8 3}$ & $\mathbf{0 . 1 7}$ \\
DT & 64.28 & 0.73 & 0.15 & $\mathbf{7 6 . 1 4}$ & $\mathbf{0 . 7 3}$ & $\mathbf{0 . 3 7}$ \\
MLP & 43.48 & 0.57 & 0.42 & 25.84 & 0.49 & 0.22 \\
\hline & & & & & &
\end{tabular}


Table 7. Exemplary classification results of UIT for selected classifiers and subjects with performance measures for both wristband and ECG.

\begin{tabular}{cccccccccccccc}
\hline \multicolumn{2}{c}{ Participant } & \multicolumn{3}{c}{$\mathbf{3}$} & & & $\mathbf{4}$ & & & $\mathbf{2 1}$ & & \multicolumn{3}{c}{$\mathbf{2 7}$} \\
\hline \multirow{2}{*}{ Model } & Device & A & F1 & F2 & A & F1 & F2 & A & F1 & F2 & A & F1 & F2 \\
\hline \multirow{2}{*}{ BN } & Wristband & 59.90 & 0.75 & 0.00 & 20.45 & 0.32 & 0.04 & 52.30 & 0.65 & 0.27 & 18.02 & 0.17 & 0.20 \\
& ECG & 51.73 & 0.59 & 0.41 & 36.57 & 0.54 & 0.00 & 77.06 & 0.87 & 0.00 & 85.49 & 0.92 & 0.51 \\
\hline \multirow{2}{*}{ KNN } & Wristband & 58.17 & 0.73 & 0.05 & 22.30 & 0.37 & 0.00 & 62.20 & 0.77 & 0.00 & 42.55 & 0.60 & 0.01 \\
& ECG & 63.41 & 0.68 & 0.58 & 42.93 & 0.59 & 0.07 & 74.56 & 0.85 & 0.04 & 78.65 & 0.86 & 0.26 \\
\hline \multirow{2}{*}{ RF } & Wristband & 57.37 & 0.73 & 0.02 & 53.16 & 0.14 & 0.68 & 63.65 & 0.78 & 0.00 & 47.43 & 0.64 & 0.04 \\
& ECG & 66.62 & 0.77 & 0.36 & 42.29 & 0.54 & 0.23 & 72.40 & 0.84 & 0.02 & 83.49 & 0.91 & 0.00 \\
\hline \multirow{2}{*}{ RT } & Wristband & 56.74 & 0.72 & 0.02 & 40.90 & 0.52 & 0.24 & 63.29 & 0.76 & 0.00 & 47.06 & 0.63 & 0.04 \\
& ECG & 67.27 & 0.78 & 0.36 & 42.29 & 0.54 & 0.23 & 74.81 & 0.86 & 0.02 & 87.49 & 0.93 & 0.00 \\
\hline \multirow{2}{*}{ DT } & Wristband & 49.60 & 0.66 & 0.00 & 34.20 & 0.04 & 0.50 & 58.09 & 0.74 & 0.00 & 33.29 & 0.43 & 0.19 \\
& ECG & 38.25 & 0.00 & 0.55 & 60.57 & 0.40 & 0.71 & 87.49 & 0.93 & 0.00 & 87.48 & 0.93 & 0.00 \\
\hline
\end{tabular}

\subsection{Discussion and Limitations}

Focusing on the results of the two devices in general, regardless of whether a UDT or UIT was performed, it is noticeable that with ECG data, slightly better results for the majority of the tested models were achieved. However, focusing on RQ2, it should be noted that with the data of the consumer device, albeit not for all models, the results are comparable and at a similar level as compared to the more intrusive medical-grade device in the in-vehicle setting. Thus, for the present application, no more complex ECG measurement would have to be applied. Instead, the much less intrusive sensor of the wrist-worn smart wearable would suffice. High accuracies (KNN: 92.13\%, RF: 91.58\%, RT: $90.02 \%$ ) as well as values for F-measure, and especially with KNN (F1: 0.95, F2: 0.83), were achieved in the UDT with data from the wristband. Concerning RQ1, this speaks for the use of physiological data, i.e., HRV, from a wristband as single data source for driver drowsiness detection with user-dependent models. Results from UDT are significantly higher than in the UIT (RQ3), which may reflect the more considerable influence of inter-driver variance that could have been better accounted for a more extensive data set. The classification of the "drowsy" class turned out to be particularly critical at UIT, which can be recognized by the low values of F2 for both devices. The maximum achieved score for F2 was 0.65 with wristband data and 0.37 with ECG data. This indicates the challenges that still need to be addressed before a robust commercial warning system can be developed. In comparison, UIT and UDT achieved high F1 values. From the customer's point of view, this is of crucial importance, as the driver does not want to be irritated by false drowsiness warnings. Moreover, data from more realistic environments has to be collected [91]. Vibrations in a real car that could influence the data recording were absent in the simulator. To be able to counteract the class imbalance, cost-sensitive classification should also be considered, in which the values of TP, FP, TN, and FN carry different weightings. Throughout the use, the system could transit from a user-independent model to a user-dependent one and adapt to the user. In terms of the ground truth, the question arises, whether it is sufficient to use drowsiness ratings that apply to 5-min intervals, or ratings at much shorter intervals are needed. During the transition to the adjacent 5-min interval, possible changes in the course of drowsiness ratings may not be reflected by the physiological signal. The applied models were used "off-the-shelf", and no hyper-parameters were tuned. The aim was to identify which standard machine learning models are suitable for the proposed classification problem. Fine-tuning of the most promising models could then increase performance further. Since the focus in this work was on a specific type of feature selection (CFSS) and class balancing, other methods should be considered and compared.

Concerning the previous studies on driver drowsiness detection using wrist-worn wearable devices, a one-to-one comparison with the proposed methodology is not possible because of the use of different data sets. The involvement of multiple sensors makes the systems more intrusive [47] and 
complex [70], whereas the proposed model only uses a wristband. In other studies, new wrist-worn wearables for recording physiological data were developed $[68,69]$. In contrast to the device used in this work, these devices are not yet ready for the market, which makes a future and large-scale use in the vehicle difficult. The use of motion sensors in the wearables to evaluate the steering behavior will be more difficult in the future [66,67], since the degree of automation will steadily increase. By using physiological data from wearables, as in the previous case as the sole data source that is continuously measured, this problem no longer exists. Except for one study [47], all others used SVM for classification. The majority achieved very high accuracies of around $98 \%$. In the present case, lower accuracies were achieved with SVM, especially with the UDT, where the wristband data only achieved an accuracy of $30 \%$, which can be due to several reasons, such as a different database, labels or feature extraction methodology. With our proposed approach with a KNN classifier, around 92\% could be achieved by using only physiological data of the wristband. The proposed methodology was implemented to show and discuss the feasibility of using solely physiological data from a wrist-worn wearable device for driver drowsiness detection. From the results, it can be seen that drowsiness can be effectively detected. The gained knowledge can serve as a starting point for further research in this area.

\section{Conclusions}

To explore the applicability of wrist-worn wearable devices for driver drowsiness detection, heart rate data from a wristband, as well as an ECG device during a simulator study with level-2 automated driving, were collected. The drowsiness level of the driver was determined based on a combination of video ratings and detected micro-sleep events. HRV features were extracted using a sliding-window approach and labeled with the corresponding drowsiness level. The performance of several machine learning classifiers was compared. Results depict that in the UDT, better results were achieved because of the reduced influence of inter-driver variance. With a value of $92.13 \%, \mathrm{KNN}$ had the highest accuracy with data from the wristband. With ECG data, $97.37 \%$ was reached with RT and RF. In the UIT, especially, the drowsy instances, were more challenging to classify. The wristband achieved $73.39 \%$ accuracy with a DS. The maximum for ECG was $78.94 \%$. In general, for both UDT and UIT, the obtained results with data from wristband and ECG are comparable. The results of our proposed methodology show that driver drowsiness detection using exclusively physiological data (HRV) from a standalone wrist-worn wearable device in combination with a machine learning model is feasible. Moreover, instead of producing a new device, the models were developed for a device available on the market that qualifies the shown method for potentially any consumer device, indicating that binary classification of drowsiness is feasible with today's technology on the consumer electronics market. Since metrics such as gyroscope or accelerometer will be more challenging to apply for driver drowsiness detection in future automated driving, thereby, our presented method would draw more attention.

The acquired knowledge can be useful for further studies in the area of driver drowsiness detection, and the advancement of vehicle-to-driver interaction concepts, furthermore, for safety-critical tasks in the area of automated driving.

\section{Future Work}

In future work, existing models improved. For feature extraction, other methods and in the present case, different increments and window sizes on the sliding window approach will be examined, and the feature space enlarged. For feature selection and class balancing, other methods than the presented ones will be considered. Since a combination of video ratings and micro-sleep events served as ground truth, in the future, the usage of driver self-ratings is planned for a comparison of classification accuracies. For an increasing generalization ability, the data set has to be enriched, and above all, with drowsy samples. Data from more users and realistic study environments will be collected. Furthermore, a prototype for real-time detection will be developed. 
Author Contributions: Conceptualization, T.K., N.S., and A.R.; methodology, T.K., N.S., and A.R.; software, T.K.; validation, T.K., N.S., and A.R.; formal analysis, T.K.; investigation, T.K.; resources, T.K., N.S., and A.R.; data curation, T.K.; writing—original draft preparation, T.K.; writing—review and editing, T.K., N.S., and A.R.; visualization, T.K.; supervision, N.S. and A.R.; project administration, T.K., N.S., and A.R. All authors have read and agreed to the published version of the manuscript.

Funding: This research received no external funding.

Acknowledgments: We applied the SDC approach for the sequence of authors.

Conflicts of Interest: The authors declare no conflict of interest.

\section{References}

1. EuroNCAP. EuroNCAP 2025 Roadmap; Technical Report; EuroNCAP: Leuven, Belgium, 2017.

2. National Highway Traffic Safety Administration; US Department of Transportation. Traffic Safety Facts Critical Reasons for Crashes Investigated in the National Motor Vehicle Crash Causation Survey; Technical Report; National Center for Statistics and Analysis (NCSA): Washington, DC, USA, 2015.

3. Tefft, B.C. Acute Sleep Deprivation and Risk of Motor Vehicle Crash Involvement. Technical Report. 2016. Available online: https://aaafoundation.org/wp-content/uploads/2017/ 12/AcuteSleepDeprivationCrashRisk.pdf (accessed on 31 December 2019).

4. Society of Automotive Engineers (SAE) International. Taxonomy and Definitions for Terms Related to Driving Automation Systems for On-Road Motor Vehicles; SAE International: Warrendale, PA, USA, 2018. [CrossRef]

5. Sahayadhas, A.; Sundaraj, K.; Murugappan, M. Detecting Driver Drowsiness Based on Sensors: A Review. Sensors 2012, 12, 16937-16953. [CrossRef]

6. Kundinger, T.; Riener, A.; Sofra, N. A Robust Drowsiness Detection Method based on Vehicle and Driver Vital Data. In Mensch und Computer 2017—Workshopband; Burghardt, M., Wimmer, R., Wolff, C., Womser-Hacker, C., Eds.; Gesellschaft für Informatik e.V.: Regensburg, Germany, 2017.

7. Poursadeghiyan, M.; Mazloumi, A.; Saraji, G.N.; Niknezhad, A.; Akbarzadeh, A.; Ebrahimi, M.H. Determination the Levels of Subjective and Observer Rating of Drowsiness and Their Associations with Facial Dynamic Changes. Iran. J. Public Health 2017, 46, 93-102. [PubMed]

8. Anund, A.; Fors, C.; Hallvig, D.; Åkerstedt, T.; Kecklund, G. Observer Rated Sleepiness and Real Road Driving: An Explorative Study. PLoS ONE 2013, 8, e0064782. [CrossRef] [PubMed]

9. Åkerstedt, T.; Gillberg, M. Subjective and objective sleepiness in the active individual. Int. J. Neurosci. 1990, 52, 29-37. [CrossRef] [PubMed]

10. Johns, M.W. A New Method for Measuring Daytime Sleepiness: The Epworth Sleepiness Scale. Sleep 1991, 14, 540-545. [CrossRef]

11. Shahid, A.; Wilkinson, K.; Marcu, S.; Shapiro, C.M. Stanford Sleepiness Scale (SSS). In Stop, that and One Hundred Other Sleep Scales; Shahid, A., Wilkinson, K., Marcu, S., Shapiro, C.M., Eds.; Springer: New York, NY, USA, 2012; pp. 369-370.

12. Monk, T.H. A visual analogue scale technique to measure global vigor and affect. Psychiatry Res. 1989, 27, 89-99. [CrossRef]

13. Weinbeer, V.; Muhr, T.; Bengler, K.; Baur, C.; Radlmayr, J.; Bill, J. Highly Automated Driving: How to Get the Driver Drowsy and How Does Drowsiness Influence Various Take-Over-Aspects? 8. Tagung Fahrerassistenz; Lehrstuhl für Fahrzeugtechnik mit TÜV SÜD Akademie: Munich, Germany, 2017.

14. Ahlstrom, C.; Fors, C.; Anund, A.; Hallvig, D. Video-based observer rated sleepiness versus self-reported subjective sleepiness in real road driving. Eur. Transp. Res. Rev. 2015, 7, 38. [CrossRef]

15. Mashko, A. Subjective Methods for the Assessment of Driver Drowsiness. Acta Polytech. CTU Proc. 2017, 12, 64. [CrossRef]

16. Knipling, R.R.; Wierwille, W.W. Vehicle-based drowsy driver detection: Current status and future prospects. In Proceedings of the IVHS AMERICA Conference Moving Toward Deployment, Atlanta, GA, USA, 17-20 April 1994.

17. Ueno, H.; Kaneda, M.; Tsukino, M. Development of drowsiness detection system. In Proceedings of the VNIS'94-1994 Vehicle Navigation and Information Systems Conference, Yokohama, Japan, 31 August-2 September 1994; pp. 15-20. 
18. Leonhardt, S.; Leicht, L.; Teichmann, D. Unobtrusive vital sign monitoring in automotive environments-A review. Sensors 2018, 18, 3080. [CrossRef]

19. Li, G.; Mao, R.; Hildre, H.; Zhang, H. Visual Attention Assessment for Expert-in-the-loop Training in a Maritime Operation Simulator. IEEE Trans. Ind. Inform. 2019, 16, 522-531. [CrossRef]

20. Sant'Ana, M.; Li, G.; Zhang, H. A Decentralized Sensor Fusion Approach to Human Fatigue Monitoring in Maritime Operations. In Proceedings of the 2019 IEEE 15th International Conference on Control and Automation (ICCA), Edinburgh, UK, 16-19 July 2019; pp. 1569-1574. [CrossRef]

21. Hu, X.; Lodewijks, G. Detecting fatigue in car drivers and aircraft pilots by using non-invasive measures: The value of differentiation of sleepiness and mental fatigue. J. Saf. Res. 2020, 72, 173-187. [CrossRef]

22. Doudou, M.; Bouabdallah, A.; Berge-Cherfaoui, V. Driver Drowsiness Measurement Technologies: Current Research, Market Solutions, and Challenges. Int. J. Intell. Transp. Syst. Res. 2019, 1-23. [CrossRef]

23. Vesselenyi, T.; Moca, S.; Rus, A.; Mitran, T.; Tătaru, B. Driver drowsiness detection using ANN image processing. IOP Conf. Ser. Mater. Sci. Eng. 2017, 252, 012097. [CrossRef]

24. Jabbar, R.; Al-Khalifa, K.; Kharbeche, M.; Alhajyaseen, W.; Jafari, M.; Jiang, S. Real-time Driver Drowsiness Detection for Android Application Using Deep Neural Networks Techniques. Procedia Comput. Sci. 2018, 130, 400-407. [CrossRef]

25. Shakeel, M.F.; Bajwa, N.A.; Anwaar, A.M.; Sohail, A.; Khan, A.; ur Rashid, H. Detecting Driver Drowsiness in Real Time Through Deep Learning Based Object Detection. In Advances in Computational Intelligence; Rojas, I., Joya, G., Catala, A., Eds.; Springer International Publishing: Cham, Switzerland, 2019; pp. 283-296.

26. Vijayan, V.; Sherly, E. Real time detection system of driver drowsiness based on representation learning using deep neural networks. J. Intell. Fuzzy Syst. 2019, 36, 1-9. [CrossRef]

27. Bamidele, A.; Kamardin, K.; Syazarin, N.; Mohd, S.; Shafi, I.; Azizan, A.; Aini, N.; Mad, H. Non-intrusive Driver Drowsiness Detection based on Face and Eye Tracking. Int J. Adv. Comput. Sci. Appl. 2019, 10, 549-569. [CrossRef]

28. SmartEye. Driver Monitoring System. Interior Sensing for Vehicle Integration. 2019. Available online: https: / smarteye.se/automotive-solutions / (accessed on 31 December 2019).

29. Edenborough, N.; Hammoud, R.; Harbach, A.; Ingold, A.; Kisacanin, B.; Malawey, P.; Newman, T.; Scharenbroch, G.; Skiver, S.; Smith, M.; et al. Driver state monitor from DELPHI. In Proceedings of the 2005 IEEE Computer Society Conference on Computer Vision and Pattern Recognition (CVPR), San Diego, CA, USA, 20-26 June 2005; Volume 2, pp. 1206-1207.

30. NoNap. NoNap Anti Sleep Alarm. 2019. Available online: http://www.thenonap.com/ (accessed on 31 December 2019).

31. Optalert. Scientifically validated Glasses-Mining. 2019. Available online: https://www.optalert.com/ explore-products / scientifically-validated-glasses-mining/ (accessed on 31 December 2019).

32. Corbett, M.A. A Drowsiness Detection System for Pilots: Optaler. Aviat. Space Environ. Med. 2009, 80, 149. [CrossRef] [PubMed]

33. Zhang, W.; Cheng, B.; Lin, Y. Driver drowsiness recognition based on computer vision technology. Tsinghua Sci. Technol. 2012, 17, 354-362. [CrossRef]

34. Trutschel, U.; Sirois, B.; Sommer, D.; Golz, M.; Edwards, D. PERCLOS: An Alertness Measure of the Past. In Proceedings of the Driving Assessment 2011: 6th International Driving Symposium on Human Factors in Driver Assessment, Training, and Vehicle Design, Lake Tahoe, CA, USA, 27-30 June 2011; pp. 172-179. [CrossRef]

35. Forsman, P.M.; Vila, B.J.; Short, R.A.; Mott, C.G.; Dongen, H.P.V. Efficient driver drowsiness detection at moderate levels of drowsiness. Accid. Anal. Prev. 2013, 50, 341-350. [CrossRef] [PubMed]

36. Ingre, M.; Åkerstedt, T.; Peters, B.; Anund, A.; Kecklund, G.; Pickles, A. Subjective sleepiness and accident risk avoiding the ecological fallacy. J. Sleep Res. 2006, 15, 142-148. [CrossRef]

37. Morris, D.M.; Pilcher, J.J.; Switzer, F.S., III. Lane heading difference: An innovative model for drowsy driving detection using retrospective analysis around curves. Accid. Anal. Prev. 2015, 80, 117-124. [CrossRef] [PubMed]

38. Friedrichs, F.; Yang, B. Drowsiness monitoring by steering and lane data based features under real driving conditions. In Proceedings of the European Signal Processing Conference, Aalborg, Denmark, 23-27 August 2010; pp. 209-213. 
39. Li, Z.; Li, S.E.; Li, R.; Cheng, B.; Shi, J. Online detection of driver fatigue using steering wheel angles for real driving conditions. Sensors 2017, 17, 495. [CrossRef] [PubMed]

40. McDonald, A.D.; Schwarz, C.; Lee, J.D.; Brown, T.L. Real-Time Detection of Drowsiness Related Lane Departures Using Steering Wheel Angle. In Proceedings of the Human Factors and Ergonomics Society Annual Meeting, Boston, MA, USA, 22-26 October 2012; Volume 56, pp. 2201-2205. [CrossRef]

41. Driver Alert System. Available online: https://www.volkswagen.co.uk/technology/car-safety/driveralert-systemn (accessed on 31 December 2019).

42. ATTENTION ASSIST: Drowsiness-Detection System Warns Drivers to Prevent Them Falling Asleep Momentarily. Available online: https://media.daimler.com $/$ marsMediaSite $/$ en $/$ instance $/$ ko.xhtml oid= 9361586 (accessed on 31 December 2019).

43. Lexus Safety System+. Available online: https://drivers.lexus.com/lexus-drivers-theme/pdf/LSS+ \%20Quick\%20Guide\%20Link.pdf (accessed on 31 December 2019).

44. Taran, S.; Bajaj, V. Drowsiness Detection Using Adaptive Hermite Decomposition and Extreme Learning Machine for Electroencephalogram Signals. IEEE Sens. J. 2018, 18, 8855-8862. [CrossRef]

45. Rundo, F.; Rinella, S.; Massimino, S.; Coco, M.; Fallica, G.; Parenti, R.; Conoci, S.; Perciavalle, V. An Innovative Deep Learning Algorithm for Drowsiness Detection from EEG Signal. Computation 2019, 7, 13. [CrossRef]

46. Budak, U.; Bajaj, V.; Akbulut, Y.; Atila, O.; Sengur, A. An Effective Hybrid Model for EEG-Based Drowsiness Detection. IEEE Sens. J. 2019, 19, 7624-7631. [CrossRef]

47. Lee, H.; Lee, J.; Shin, M. Using Wearable ECG/PPG Sensors for Driver Drowsiness Detection Based on Distinguishable Pattern of Recurrence Plots. Electronics 2019, 8, 192. [CrossRef]

48. Gromer, M.; Salb, D.; Walzer, T.; Madrid, N.M.; Seepold, R. ECG sensor for detection of driver's drowsiness. Procedia Comput. Sci. 2019, 159, 1938-1946. [CrossRef]

49. Babaeian, M.; Mozumdar, M. Driver Drowsiness Detection Algorithms Using Electrocardiogram Data Analysis. In Proceedings of the 2019 IEEE 9th Annual Computing and Communication Workshop and Conference (CCWC), Las Vegas, NV, USA, 7-9 January 2019; pp. 0001-0006.

50. Zheng, W.; Gao, K.; Li, G.; Liu, W.; Liu, C.; Liu, J.; Wang, G.; Lu, B. Vigilance Estimation Using a Wearable EOG Device in Real Driving Environment. IEEE Trans. Intell. Transp. Syst. 2019, 21, 1-15. [CrossRef]

51. Barua, S.; Ahmed, M.U.; Ahlström, C.; Begum, S. Automatic driver sleepiness detection using EEG, EOG and contextual information. Expert Syst. Appl. 2019, 115, 121-135. [CrossRef]

52. Hu, S.; Zheng, G. Driver drowsiness detection with eyelid related parameters by Support Vector Machine. Expert Syst. Appl. 2009, 36, 7651-7658. [CrossRef]

53. Mahmoodi, M.; Nahvi, A. Driver drowsiness detection based on classification of surface electromyography features in a driving simulator. Proc. Inst. Mech. Eng. Part H J. Eng. Med. 2019, 233, 395-406. [CrossRef]

54. Fu, R.; Wang, H. Detection of Driving Fatigue by using noncontact EMG and ECG signals measurement system. Int. J. Neural Syst. 2014, 24, 1450006. [CrossRef] [PubMed]

55. Wörle, J.; Metz, B.; Thiele, C.; Weller, G. Detecting sleep in drivers during highly automated driving: The potential of physiological parameters. IET Intell. Transp. Syst. 2019, 13, 1241-1248. [CrossRef]

56. Ramesh, M.V.; Nair, A.K.; Kunnathu, A.T. Real-Time Automated Multiplexed Sensor System for Driver Drowsiness Detection. In Proceedings of the 2011 7th International Conference on Wireless Communications, Networking and Mobile Computing, Wuhan, China, 23-25 September 2011; pp. 1-4. [CrossRef]

57. Rahim, H.; Dalimi, A.; Jaafar, H. Detecting Drowsy Driver Using Pulse Sensor. J. Teknol. 2015, 73, 5-8. [CrossRef]

58. Jung, S.; Shin, H.; Chung, W. Driver fatigue and drowsiness monitoring system with embedded electrocardiogram sensor on steering wheel. IET Intell. Transp. Syst. 2014, 8, 43-50. [CrossRef]

59. Solaz, J.; Laparra-Hernández, J.; Bande, D.; Rodríguez, N.; Veleff, S.; Gerpe, J.; Medina, E. Drowsiness Detection Based on the Analysis of Breathing Rate Obtained from Real-time Image Recognition. Transp. Res. Procedia 2016, 14, 3867-3876. [CrossRef]

60. Final Report Summary-HARKEN (Heart and Respiration in-Car Embedded Nonintrusive Sensors)। Report Summary I HARKEN I FP7 I CORDIS I European Commission. 2014. Available online: https: / / cordis. europa.eu/project/rcn/103870/reporting/en (accessed on 31 December 2019).

61. Creative Mode. STEER: Wearable Device That Will Not Let You Fall Asleep. 2019. Available online: https:/ / www.kickstarter.com/projects/creativemode/steer-you-will-never-fall-asleepwhile-driving?lang=en (accessed on 31 December 2019). 
62. StopSleep. Anti-Sleep Alarm. 2019. Available online: https://www.stopsleep.co.uk/ (accessed on 31 December 2019).

63. Neurocom. Driver Vigilance Telemetric Control System-VIGITON. 2019. Available online: http://www. neurocom.ru/en2/product/vigiton.html (accessed on 31 December 2019).

64. Strategy Analytics. Global Smartwatch Vendor Market Share by Region: Q4 2018. 2015. Available online: https:/ / www.strategyanalytics.com/access-services / devices/wearables / market-data / report-detail/global-smartwatch-vendor-market-share-by-region-q4-2018 (accessed on 31 December 2019).

65. Georgiou, K.; Larentzakis, A.V.; Khamis, N.N.; Alsuhaibani, G.I.; Alaska, Y.A.; Giallafos, E.J. Can Wearable Devices Accurately Measure Heart Rate Variability? A Systematic Review. Folia Med. 2018, 60, 7-20. [CrossRef]

66. Lee, B.; Lee, B.; Chung, W. Standalone Wearable Driver Drowsiness Detection System in a Smartwatch. IEEE Sens. J. 2016, 16, 5444-5451. [CrossRef]

67. Lee, B.L.; Lee, B.G.; Li, G.; Chung, W.Y. Wearable Driver Drowsiness Detection System Based on Smartwatch. In Proceedings of the Korea Institute of Signal Processing and Systems (KISPS) Fall Conference, Korea, Japan, 21-22 November 2014; Volume 15, pp. 134-146.

68. Leng, L.B.; Giin, L.B.; Chung, W. Wearable driver drowsiness detection system based on biomedical and motion sensors. In Proceedings of the 2015 IEEE SENSORS, Busan, Korea, 1-4 November 2015. [CrossRef]

69. Choi, M.; Koo, G.; Seo, M.; Kim, S.W. Wearable Device-Based System to Monitor a Driver's Stress, Fatigue, and Drowsiness. IEEE Trans. Instrum. Meas. 2018, 67, 634-645. [CrossRef]

70. Li, Q.; Wu, J.; Kim, S.D.; Kim, C.G. Hybrid Driver Fatigue Detection System Based on Data Fusion with Wearable Sensor Devices. Available online: https://www.semanticscholar.org/paper/Hybrid-DriverFatigue-Detection-System-Based-on-Li-Wu/90da9d40baa5172d962930d838e5ea040f463bad (accessed on 14 February 2020).

71. Kundinger, T.; Riener, A.; Sofra, N.; Weigl, K. Drowsiness Detection and Warning in Manual and Automated Driving: Results from Subjective Evaluation. In Proceedings of the 10th International Conference on Automotive User Interfaces and Interactive Vehicular Applications, AutomotiveUI '18, Toronto, ON, Canada, 23-25 September 2018; pp. 229-236. [CrossRef]

72. Sleep Health Foundation. Sleep Needs Across the Lifespan. 2015. Available online: http:/ / www.sleephealthfoundation.org.au/files/pdfs/Sleep-Needs-Across-Lifespan.pdf (accessed on 31 December 2019).

73. Support, E. Recent Publications Citing the E4 wristband. 2018. Available online: https://support. empatica.com/hc/en-us/articles/115002540543-Recent-Publications-citing-the-E4-wristband- (accessed on 31 December 2019).

74. McCarthy, C.; Pradhan, N.; Redpath, C.; Adler, A. Validation of the Empatica E4 wristband. In Proceedings of the 2016 IEEE EMBS International Student Conference (ISC), Ottawa, ON, Canada, 29-31 May 2016; pp. 1-4. [CrossRef]

75. Corporation, B. Bittium Faros Waterproof ECG Devices. 2019. Available online: https://support. empatica.com/hc/en-us/articles/115002540543-Recent-Publications-citing-the-E4-wristband- (accessed on 31 December 2019).

76. Vicente, J.; Laguna, P.; Bartra, A.; Bailón, R. Drowsiness detection using heart rate variability. Med. Biol. Eng. Comput. 2016, 54, 927-937. [CrossRef]

77. Michail, E.; Kokonozi, A.; Chouvarda, I.; Maglaveras, N. EEG and HRV markers of sleepiness and loss of control during car driving. In Proceedings of the 2008 30th Annual International Conference of the IEEE Engineering in Medicine and Biology Society, Vancouver, BC, Canada, 20-25 August 2008; pp. 2566-2569. [CrossRef]

78. Malik, M.; Camm, A.; Bigger, J.; Breithardt, G.; Cerutti, S.; Cohen, R.; Coumel, P.; Fallen, E.; Kennedy, H.; Kleiger, R.; et al. Heart rate variability. Standards of measurement, physiological interpretation, and clinical use. Eur. Heart J. 1996, 17, 354-381. [CrossRef]

79. Lee, I.; Lau, P.; Chua, E.C.P.; Gooley, J.J.; Tan, W.Q.; Yeo, S.C.; Puvanendran, K.; Mien, I.H. Heart Rate Variability Can Be Used to Estimate Sleepiness-related Decrements in Psychomotor Vigilance during Total Sleep Deprivation. Sleep 2012, 35, 325-334. [CrossRef] 
80. Sandberg, D. The performance of driver sleepiness indicators as a function of interval length. In Proceedings of the 2011 14th International IEEE Conference on Intelligent Transportation Systems (ITSC), Washington, DC, USA, 5-7 October 2011; pp. 1735-1740. [CrossRef]

81. Landis, J.R.; Koch, G.G. The Measurement of Observer Agreement for Categorical Data; Technical Report 1; International Biometric Society: Washington, DC, USA, 1977.

82. Tarvainen, M.P.; Niskanen, J.P.; Lipponen, J.A.; Ranta-aho, P.O.; Karjalainen, P.A. Kubios HRV-Heart rate variability analysis software. Comput. Methods Programs Biomed. 2014, 113, 210-220. [CrossRef] [PubMed]

83. Empatica Support. E4 Data-IBI Expected Signal. 2020. Available online: https://support.empatica.com/ hc/en-us/articles/360030058011-E4-data-IBI-expected-signal (accessed on 31 January 2020).

84. Li, G.; Chung, W.Y. Detection of Driver Drowsiness Using Wavelet Analysis of Heart Rate Variability and a Support Vector Machine Classifier. Sensors 2013, 13, 16494-16511. [CrossRef]

85. Shirmohammadi, S.; Barbe, K.; Grimaldi, D.; Rapuano, S.; Grassini, S. Instrumentation and measurement in medical, biomedical, and healthcare systems. IEEE Instrum. Meas. Mag. 2016, 19, 6-12. [CrossRef]

86. Kohavi, R. A Study of Cross-validation and Bootstrap for Accuracy Estimation and Model Selection. In Proceedings of the 14th International Joint Conference on Artificial Intelligence, IJCAI'95, Adelaide, Australia, 10-14 December 2001; Morgan Kaufmann Publishers Inc.: San Francisco, CA, USA, 1995; Volume 2, pp. 1137-1143.

87. Chawla, N.; Bowyer, K.; Hall, L.; Kegelmeyer, W. SMOTE: Synthetic Minority Over-sampling Technique. J. Artif. Intell. Res. 2002, 16, 321-357. [CrossRef]

88. Hall, M.A. Correlation-Based Feature Subset Selection for Machine Learning. Ph.D. Thesis, University of Waikato, Hamilton, NZ, USA, 1998.

89. Branco, P.; Torgo, L.; Ribeiro, R.P. A Survey of Predictive Modeling on Imbalanced Domains. ACM Comput. Surv. 2016, 49. [CrossRef]

90. Hall, M.; Frank, E.; Holmes, G.; Pfahringer, B.; Reutemann, P.; Witten, I.H. The WEKA Data Mining Software: An Update. SIGKDD Explor. 2009, 11, 10-18. [CrossRef]

91. Persson, A.; Jonasson, H.; Fredriksson, I.; Wiklund, U.; Ahlström, C. Heart Rate Variability for Driver Sleepiness Classification in Real Road Driving Conditions. In Proceedings of the 2019 41st Annual International Conference of the IEEE Engineering in Medicine and Biology Society (EMBC), Berlin, Germany, 23-27 July 2019; pp. 6537-6540. [CrossRef]

(C) 2020 by the authors. Licensee MDPI, Basel, Switzerland. This article is an open access article distributed under the terms and conditions of the Creative Commons Attribution (CC BY) license (http://creativecommons.org/licenses/by/4.0/). 